\title{
Потенциальная чувствительность
} к метформину у больных диабетом, имеющих сопутствующую онкопатологию и без таковой: фармакогенетический анализ

\begin{abstract}
Пациенты и методы. Обследовано 156 постменопаузальных женщин, среди которых были 32 больные сахарным диабетом 2-го типа (СД2), $64-$ с сочетанием СД2 и нелеченой злокачественной опухоли (по большей части рак толстой кишки, молочной железы или эндометрия), 23 - с онкологическим заболеванием без диабета и 37 здоровых. Изучено носительство 8 генетических полиморфизмов: четырех, обозначенных как «стандартные» (C), поскольку в отношении них ранее имелись сведения о связи преимущественно с метаболическим ответом на противодиабетический препарат из группы бигуанидов метформин, и еще четырех, причисленных к «ассоциированным» (А). Результаты. Межжду больными СД2, не страдающими или страдающими злокачественными новообразованиями, достоверных различий в носительстве C-вариантов найдено не было. Потенциальную склонность к ответу на метформин у онкологических больных без диабета наилучшим образом характеризовало носительство вариантов генов STK11, а исследование у них же полиморфизмов OCT1_rs622342 и OCT1_R61C обнаружило противоположные по направленности результаты. Страдающие впервые выявленным СД2 носители потенциально метформинпозитивных полиморфных вариантов OCT1_R61C и OCT1_rs622342 характеризовались более выраженной инсулинорезистентностью, в то время как аналогичного характера подгруппа носителей полиморфизмов генов STK11 и C11orf65 - умеренно сниженной эстрадиолемией. Выводы. Больные СД2, имеющие и не имеющие злокачественные новообразования, по генетическим критериям потенциальной чувствительности $\kappa$ метформину между собой отличаются меньше, чем от онкологических больных без диабета. Предиктивная эффективность С-критериев может быть повышена путем их сочетания с оценкой А-полиморфизмов и некоторых гормонально-метаболических параметров.

Ключевые слова: диабет, рак, метформин, фармакогенетика/фармакогеномика.
\end{abstract}

(Вестник РАМН. 2013; 12: 58-63)

\section{Введение}

Население немалого числа развитых и развивающихся стран, по статистическим данным, «стареет» и склонно к полноте и развитию диабета, что приобрело характер эпидемии [1] и, как полагают, может оказывать влияние на частоту развития и клиническое течение онкологических заболеваний. Связь сахарного диабета (СД) с онкопатологией варьирует в зависимости от типа диабета, пола больных, локализации опу- холи и ряда других факторов, включая использование антидиабетических препаратов [2, 3]. В лечении СД 2-го типа (СД2) значимое место занимают бигуаниды, в настоящее время представленные метформином. Роль последнего как модификатора общей и онкологической заболеваемости и смертности пока окончательно не выяснена, что может быть обусловлено селективностью его действия по отношению и к отдельным индивидуумам, и к вовлекаемым метаболическим и иным процессам, и к тканям-мишеням [3, 4].

\section{L.M. Berstein, A.G. Iyevleva, D.A. Vasilyev, I.M. Kovalenko, E.N. Imyanitov \\ N.N. Petrov Research Institute of Oncology, St. Petersburg, Russian Federation}

\section{Potential Sensitivity to Metformin of the Diabetics Suffering and Not Suffering with Cancer: a Pharmacogenetic Study}

The group (totally156 postmenopausal women) used for the study of 'standard' (S) and 'associated' (A) genetic markers of potential sensitivity to metformin (MF) consisted of 37 healthy females, 32 - with diabetes (DM) without cancer, 64 cancer patients with DM, and 23 cancer patients without DM. No significant difference in carrying of S-polymorphisms was found between DM patients without and with cancer. In cancer patients without DM most characteristic data regarding potential MF-response were detected with polymorphisms of STK11 gene while data on OCT1_rs622342 and OCT1_R61C variants showed opposite trends. In regard of A-markers, the tendency to the more often finding of GC genotype of OLR1_G501C in DM patients carrying 'MF-positive' variant of OCT1_R61C deserves to be underlined. In patients with new-onset diabetes who carried S-markers of potential response to MF higher insulin resistance (OCT1_R61C and OCT1_rs622342) as well as lower estradiolemia (STK11 and C11orf65) were discovered. Thus, according to genetic S-criteria of sensitivity to MF, DM patients with and without cancer differ in lesser degree than they differ from cancer patients without DM. It can not be excluded, that The efficiency of such criteria might be increased due to combination with A-markers and certain hormonal-metabolic indices.

Key words: diabetes, cancer, metformin, pharmacogenetics / pharmacogenomics.

(Vestnik Rossiiskoi Akademii Meditsinskikh Nauk - Annals of the Russian Academy of Medical Sciences. 2013; 12: 58-63) 
Индивидуальные (person to person distinctions) особенности терапевтического воздействия метформина достаточно хорошо известны из клинической практики, что подтверждается, в частности, давно сформировавшимся мнением о большей эффективности этого препарата как средства лечения СД2 у людей с избыточной массой тела, хотя иногда приводятся примеры и обратного характера [5]. С другой стороны, по отношению к возможной вариабельности действия метформина в последние годы все чаще говорят о значении его концентрации в крови и скорости / интенсивности утилизации в тканях, существенное влияние на которые могут оказывать характерологические свойства генома, являющиеся предметом фармакогеномики или фармакогенетики.

Исследования, где фармакогенетический подход к оценке вариабельности в действии метформина использовался в условиях клиники, до настоящего времени выполнялись нечасто и по большей части концентрировались вокруг полиморфных вариантов транспортера 1 органических катионов (organic cation transporter 1, ОСТ1) [6]. Метформин принадлежит к числу субстратов ОСТ1 белка, интенсивно экспрессирующегося в печени. У нокаутных по ОCT1 мышей снижен захват метформина в этом органе и некоторых других тканях. Это привело к идее о наличии взаимосвязи между носительством отдельных полиморфизмов ОСТ1 и непостоянством ответа на метформин, которая была подтверждена в нескольких исследованиях [6, 7]. Помимо гена ОCT1, связь с метаболическим ответом на метформин выявлена при диабете у носителей полиморфных маркеров гена C11orf65 (rs 11212617), локализующегося недалеко от гена атаксии-телеангиэктазии $A T M$ [8], и у больных поликистозом яичников при анализе полиморфизмов гена $S T K 11$ [9], кодирующего онкосупрессорный белок LKB1. При выпадении функции последнего недостаточно активируется аденозинмонофосфаткиназа (АМРК), что в ряде ситуаций удается скорректировать назначением метформина $[1,3,4]$.

Следует упомянуть также о вероятности существования полиморфных вариантов, которые могут быть ассоциированы (А-полиморфизмы) с последствиями носительства более известных и только что перечисленных (условно стандартных, С) генетических маркеров ответа на метформин.

Цель исследования: впервые сравнить частоту носительства полиморфизмов С-генов, указывающих на потенциальную возможность позитивного или ослабленного эффекта метформина [6-9], у постменопаузальных больных СД2, имеющих или, напротив, не имеющих онкологическое заболевание. Параллельно проводилась оценка того, в какой степени предположительные генетические А-маркеры, сопряженные с риском развития нарушенной толерантности к глюкозе, метаболического синдрома, некоторых злокачественных новообразований [10-12], комбинируются с упомянутыми С-маркерами, и имеются ли какие-либо особенности гормонально-метаболического статуса у женщин с различным индивидуальным ответом на метформин по данным проведенного генетического анализа.

\section{Пациенты и методы}

\section{Участники исследования}

В исследовании участвовали 156 женщин в возрасте от 43 до 88 лет с длительностью менопаузы не менее 1 года. Обследуемые были разделены на группы: больные
СД2 без онкологических заболеваний $(n=32$, средний возраст 62,0土1,5 года); больные с сочетанием СД2 и недавно выявленного злокачественного новообразования - главным образом рака толстой кишки, молочной железы или эндометрия ( $n=64$, средний возраст $62,5 \pm 1,1$ года); больные с онкологическим заболеванием без признаков СД2 ( $n=23$, средний возраст 59,7 2,0 года)

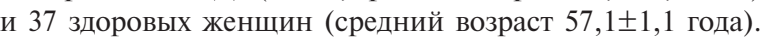
В объединенной группе больных диабетом $(n=96)$ в 65 случаях СД2 был выявлен впервые, а в 31 наблюдении больные СД2 получали тот или иной вариант антидиабетической терапии (24 пациентки с сочетанием диабета и онкозаболевания и 7 человек только с диабетом). В той же объединенной группе больных СД2 у 35 женщин имелись близкие родственники, страдавшие диабетом. Никто из онкологических больных никакой специфической противоопухолевой терапии до момента обследования не получал.

В ходе работы изучены полиморфизмы «стандартных» (C) генов, в отношении которых имеются сведения о связи с ответом на метформин [6-9]. К этой группе отнесены полиморфные варианты генов OCT1 (R61C_ rs 12208357 и интронный вариант $A>C$ rs622342), гена серин-треониновой киназы В1 STK11/LKB1 (rs8111699) и гена C11orf65 (rs 11212617), локализующегося, как упомянуто выше, в том же локусе, что и ген $A T M$. Помимо этих четырех полиморфизмов исследовано такое же число полиморфных вариантов генов, обозначенных выше как «ассоциированные» (А). В эту группу вошли полиморфизмы сопряженного с процессом репарации ДНК гена 8-оксигуанин-ДНК-гликозилазы OGG1Ser326Cys ( $r s 1052134)$, гена рецепторов окисленных липопротеинов низкой плотности OLR1_G501C (rs 1053646), гена рецепторов лептина LEPR_Gln223Arg (rs 1137101) и гена половые гормоны связываюшего глобулина $S H B G \_T>C$ (rs6257).

\section{Методы исследования}

Источником ДНК, выделяемой соль-хлороформным методом, служили лейкоциты периферической крови, пробы которой отбирались после ночного голодания. Генотипирование образцов проводили при помощи аллель-специфической полимеразной цепной реакции (ПЦР) в режиме реального времени на приборе iQ iCycler (Bio-Rad, CША) с использованием интеркалирующего красителя SYBR Green I. Праймеры, их температуры отжига и длины синтезированных фрагментов для анализа полиморфизма соответствующих генов приведены в табл. 1. ПЦР-амплификация проходила в объеме 20 мкл. В состав реакционной смеси входили: 1 ед. акт. «hot-start» Таq-полимеразы, однократный ПЦР-буфер, 50 нг ДНК, 1,5-3,0 мМ $\mathrm{MgCl}_{2}$, по 200 мкМ каждого из дезоксинуклеотидтрифосфатов (dATP, dCTP, dGTP, dTTP), 100 нМ каждого праймера, 0,2 мкл 20-кратного раствора SYBR-Green I. Реакция начиналась с фазы активации Таq-полимеразы $\left(95^{\circ} \mathrm{C}, 7\right.$ мин). Последующие 45 циклов ПЦР состояли из фаз денатурации $\left(95^{\circ} \mathrm{C}, 30 \mathrm{c}\right)$, отжига $\left(60-66^{\circ} \mathrm{C}, 60 \mathrm{c}\right)$ и элонгации $\left(72{ }^{\circ} \mathrm{C}, 60 \mathrm{c}\right)$.

В дополнение у 65 больных СД2 (из них $40-c$ недавно выявленным онкологическим заболеванием и 25 - без онкопатологии) до начала какой-либо антидиабетической терапии и после 10-12-часового ночного голодания, помимо определения гликемии и содержания гликилированного гемоглобина, производили оценку липид-, инсулини эстрадиолемии в зависимости от параметра энзимоколориметрическим или иммуноферментным методом и рассчитывали индекс инсулинорезистентности (HOMA-IR) по формуле Matthews и соавт. [13]. 
Таблица 1. Информация об использованных в работе последовательностях праймеров, температурах их отжига и длине синтезированных фрагментов

\begin{tabular}{|c|c|c|c|c|}
\hline Ген & $\begin{array}{c}\text { Однонуклеотидные } \\
\text { полиморфизмы }\end{array}$ & 5'-3'-последовательность праймеров & $\begin{array}{c}\text { Длина } \\
\text { фрагмента }\end{array}$ & $\begin{array}{c}\text { Температура } \\
\text { отжига }\end{array}$ \\
\hline \multirow[t]{3}{*}{ OCT1 } & \multirow[t]{3}{*}{$\mathrm{R} 61 \mathrm{C}$} & AGG GCT CCA GCC ACA GCG (OCT1-61-C) & \multirow[t]{3}{*}{120 п.о. } & \multirow[t]{3}{*}{$66^{\circ} \mathrm{C}$} \\
\hline & & AGG GCT CCA GCC ACA GCA (OCT1-61-T) & & \\
\hline & & CTG CTG TCG GCT GCC TTT G (OCT1-61-F) & & \\
\hline \multirow[t]{3}{*}{ OCT1 } & \multirow[t]{3}{*}{$A>C($ rs 622342$)$} & AGA TTG TTA GAT CTA TGT ATT TG (OCT1-A) & \multirow[t]{3}{*}{153 п.о. } & \multirow[t]{3}{*}{$60{ }^{\circ} \mathrm{C}$} \\
\hline & & AGA TTG TTA GAT CTA TGT ATT GG (OCT1-C) & & \\
\hline & & GAA AGA CAG AGA GAA TCA GTG (OCT1-com) & & \\
\hline \multirow[t]{3}{*}{ STK11 } & \multirow[t]{3}{*}{$C>G($ rs8111699) } & TGT GAG AGT GAG CCC CCT (STK11-C) & \multirow[t]{3}{*}{179 п.о. } & \multirow[t]{3}{*}{$65^{\circ} \mathrm{C}$} \\
\hline & & TGT GAG AGT GAG CCC CGT (STK11-G) & & \\
\hline & & CCT CCC TGC CTC CGT GTT (STK11-R) & & \\
\hline \multirow[t]{3}{*}{ C11orf65 } & \multirow[t]{3}{*}{$G>\operatorname{Trs} 11212617$} & TAC AAA GGG CAG ATC AGA GAC (C11orf65-G) & \multirow[t]{3}{*}{160 п.о. } & \multirow[t]{3}{*}{$60{ }^{\circ} \mathrm{C}$} \\
\hline & & TAC AAA GGG CAG ATC AGA GAA (C11orf65-T) & & \\
\hline & & TGC GTG GAG TCA GAG TCTA A (C11orf65-R) & & \\
\hline \multirow[t]{3}{*}{$O G G 1$} & \multirow[t]{3}{*}{ Ser326Cys } & TGC CGA CCT GCG CCA ATC (OGG1-G) & \multirow[t]{3}{*}{89 п.о. } & \multirow[t]{3}{*}{$65^{\circ} \mathrm{C}$} \\
\hline & & TGC CGA CCT GCG CCA ATG (OGG1-C) & & \\
\hline & & GGT GCC CCA TCT AGC CTT (OGG1-R) & & \\
\hline \multirow[t]{3}{*}{ OLR1 } & \multirow[t]{3}{*}{ G501C } & GCTCATTTAACTGGGAAAAGA (OLR1-501-G) & \multirow[t]{3}{*}{164 п.о. } & \multirow[t]{3}{*}{$60{ }^{\circ} \mathrm{C}$} \\
\hline & & GCTCATTTAACTGGGAAAACA (OLR1-501-C) & & \\
\hline & & ATTCCTCCAGTGACAGTTTA (OLR1-501-R) & & \\
\hline \multirow[t]{3}{*}{$L E P R$} & \multirow[t]{3}{*}{ Gln223Arg } & AAC TGA CAT TAG AGG TGA CC (LEPR-223-G) & \multirow[t]{3}{*}{122 п.о. } & \multirow[t]{3}{*}{$63^{\circ} \mathrm{C}$} \\
\hline & & AAC TGA CAT TAG AGG TGA CT (LEPR-223-A) & & \\
\hline & & ATG TTG TGA ATG TCT TGT GC (LEPR-223-com) & & \\
\hline \multirow[t]{3}{*}{$S H B G$} & \multirow[t]{3}{*}{$T>C(r s 6257)$} & TCC CTA CTC AGC TTT GTT TGT (SHBG-6257-T) & \multirow[t]{3}{*}{177 п.о. } & \multirow[t]{3}{*}{$65^{\circ} \mathrm{C}$} \\
\hline & & TCC CTA CTC AGC TTT GTT TGC (SHBG-6257-C) & & \\
\hline & & AGA GGG CAG AAC CAG GGG A (SHBG-6257-R) & & \\
\hline
\end{tabular}

\section{Статистическая обработка данных}

Статистический анализ результатов осуществляли с помощью пакета прикладных программ SigmaPlot for Windows (Systat Software, США) и Statistica 8.0 (Statsoft Inc., США). Тест на гетерогенность результатов проводили, сравнивая распределение генотипов по каждому полиморфизму между группами с помощью $\chi^{2}$-критерия Пирсона ( $\chi^{2}$ с одной степенью свободы). Сравнение гормонально-метаболических показателей в отдельных группах $(\mathrm{M} \pm \mathrm{m})$ осуществляли на основании $t$-критерия Стьюдента. Критический уровень значимости при проверке статистических гипотез принимали равным 0,05 .

\section{Результаты}

Частота обнаружения полиморфных вариантов сопряженных с потенциальным ответом на метформин генов, которые обозначены выше как «стандартные» (C), представлена в табл. 2. Между больными СД2, не страдающими или страдающими злокачественными новообразованиями, различий в носительстве вариантов этих генов найдено не было, хотя по носительству прежде всего полиморфизма $O C T 1 \_R 61 C$ обе подгруппы больных диабетом от онкологических больных без диабета отличались весьма заметно. С другой стороны, тенденция к более частому по сравнению со здоровыми женщинами носительству метформинпозитивного варианта C11orf65 была отмечена только у больных СД2 без онкопатологии $\left(\chi^{2}=2,18\right)$. Потенциальную склонность к ответу на метформин у онкологических больных без диабета наилучшим образом характеризовало носительство вариантов генов STK11 и OCT1_rs622342 (напротив, частое носительство в этой группе генотипов CT и $T T$ OCT1_R61C указывало на возможность противоположного по направленности, т.е. ослабленного, ответа на препарат; значения $\chi^{2}$ см. в табл. 2). Наконец, у больных семейным СД2 по сравнению со здоровыми женщинами и онкологическими больными без диабета маркером потенциального снижения чувствительности к метформину явилось редкое носительство генотипа $G G \operatorname{STK11}\left(\chi^{2}=2,89\right.$ и 3,36 ; $\mathrm{p}=0,09$ и 0,07 , соответственно).

При анализе полиморфных маркеров, обозначенных как «ассоциированные» (А), достоверные различия были зарегистрированы только при сравнении данных у больных СД2 в комбинации с онкопатологией, с одной стороны, и онкологических больных без диабета - с другой. В частности, в первой из этих групп по сравнению со второй носительство варианта $A G$ гена рецепторов лептина было более редким $(46,9 \pm 6,1$ против $78,3 \pm 8,4 \%$, $\left.\chi^{2}=6,74 ; p=0,01\right)$, а носительство генотипа $T C$ гена половые гормоны связывающего глобулина (rs6257) - более частым $\left(17,2 \pm 4,7\right.$ против $\left.0 \%, \chi^{2}=4,53 ; p=0,03\right)$. Оценка А-полиморфизмов в подгруппах женшин-носительниц «стандартных» генотипов, характеризующихся потенциально различающимся ответом на метформин, продемонстрировала тенденцию к более частому носительству варианта $G C$ гена рецепторов окисленных липопротеинов OLR1_G501C в объединенной группе больных СД2 $\left(\chi^{2}=2,87 ; p=0,09\right)$ и группе диабетиков среди онкологических больных $\left(\chi^{2}=2,59 ; p=0,11\right)$, носящих метформинпозитивный вариант OCT1 R61C посравнениюс метформиннегативным вариантом того же гена (табл. 3). Похожее заключение может быть сделано в отношении варианта $T C$ гена половые гормоны связывающего глобулина rs6257 применительно к его комбинации с генотипами $\mathrm{AC}$ и CC OCT1_rs622342 $\left(\chi^{2}=3,04\right.$ и 2,$17 ; p=0,08$ и 0,14 , соответственно). В то же время в объединенной группе 
Таблица 2. Частота (\%) обнаружения у обследуемых женщин «стандартных» (С) генотипов, указывающих на потенциальную направленность ответа на метформин

\begin{tabular}{|c|c|c|c|c|}
\hline Группы женщин & $\begin{array}{c}\text { OCT1 R61C } \\
(C T+T T)^{1}\end{array}$ & $\begin{array}{c}\text { OCT1 rs622342 } \\
(C C)^{1}\end{array}$ & $\begin{array}{l}\text { STK11 } \\
(G G)^{2}\end{array}$ & $\begin{array}{c}\text { C11orf65 near ATM } \\
(C C)^{2}\end{array}$ \\
\hline Больные СД2 без онкопатологии $(n=32)$ & $9,4 \pm 5,1^{3}$ & $15,6 \pm 6,4$ & $12,5 \pm 5,9$ & $31,3 \pm 8,1$ \\
\hline Больные СД2 в сочетании с онкологическим заболеванием $(n=64)$ & $12,5 \pm 4,1^{3}$ & $14,1 \pm 4,2$ & $14,1 \pm 4,2$ & $20,3 \pm 5,0$ \\
\hline Объединенная группа больных СД2 ( $n=96)$ & $11,5 \pm 3,2^{3}$ & $14,6 \pm 3,4$ & $13,5 \pm 3,5$ & $24,0 \pm 4,3$ \\
\hline Больные семейной формой СД2 $(n=35)$ & $17,1 \pm 6,3$ & $11,4 \pm 5,3$ & $5,7 \pm 4,0$ & $22,2 \pm 7,0$ \\
\hline Онкологические больные, не страдающие СД2 $(n=23)$ & $34,8 \pm 9,9$ & $4,3 \pm 3,9$ & $21,7 \pm 4,2$ & $21,7 \pm 4,2$ \\
\hline Здоровые $(n=37)$ & $18,9 \pm 6,3$ & $16,2 \pm 6,0$ & $18,9 \pm 6,4$ & $16,2 \pm 6,0$ \\
\hline
\end{tabular}

Примечание. СД2 - сахарный диабет 2 -го типа или нарушенная толерантность к глюкозе; $n-$ число наблюдений; ${ }^{1}-$ генотипы-маркеры потенциально ослабленного ответа на метформин; ${ }^{2}-$ генотипы-маркеры потенциального позитивного ответа на метформин; 3 - отличие от группы онкологических больных, не страдающих диабетом, достоверно $\left(\chi^{2} 5,40-7,52 ; p<0,01-0,02\right)$.

Таблица 3. Распределение (\%) «ассоциированных» (А) генотипов в группах постменопаузальных больных диабетом - носительниц генетических С-маркеров ответа на метформин

\begin{tabular}{|c|c|c|c|c|c|c|c|}
\hline \multirow[t]{2}{*}{ А-генотипы } & \multirow[t]{2}{*}{$\begin{array}{l}\text { Групाыы } \\
\text { больных }\end{array}$} & \multicolumn{6}{|c|}{$\begin{array}{c}\text { Варианты «стандартных» генотипов, ассоциированные с потенциально позитивным (+) } \\
\text { или негативным (-) ответом на метформин }\end{array}$} \\
\hline & & $\begin{array}{c}\text { OCT1 R61C (+) } \\
C C\end{array}$ & $\begin{array}{c}\text { OCT1 R61C (-) } \\
C T+T T\end{array}$ & $\begin{array}{c}\text { OCTrs622342 (+) } \\
A C+A A\end{array}$ & $\begin{array}{c}\text { OCTrs622342 (-) } \\
\text { CC }\end{array}$ & $\begin{array}{c}\text { C110rf65 (+) } \\
\text { CC }\end{array}$ & $\begin{array}{c}\text { C110rf65 (-) } \\
A A\end{array}$ \\
\hline \multirow[t]{2}{*}{$\begin{array}{l}\text { OGG1 Ser326Cys } \\
(C G+G G)\end{array}$} & $\begin{array}{c}\text { СД2 + ОП } \\
(n=64)\end{array}$ & $\begin{array}{c}31,6 \pm 6,2 \\
(n=56)\end{array}$ & $\begin{array}{c}25,0 \pm 11,3 \\
(n=8)\end{array}$ & $\begin{array}{c}30,3 \pm 6,1 \\
(n=55)\end{array}$ & $\begin{array}{c}33,3 \pm 15,7 \\
(n=9)\end{array}$ & $\begin{array}{l}8,3 \pm 7,5 \\
(n=12)^{3}\end{array}$ & $\begin{array}{c}36,5 \pm 6,9 \\
(n=52)\end{array}$ \\
\hline & $\begin{array}{c}\text { СД2, все } \\
(n=96)\end{array}$ & $\begin{array}{c}38,4 \pm 5,2 \\
(n=85)\end{array}$ & $\begin{array}{c}18,2 \pm 11,5 \\
(n=11)\end{array}$ & $\begin{array}{c}32,5 \pm 5,2 \\
(n=82)\end{array}$ & $\begin{array}{c}57,1 \pm 13,2 \\
(n=14)\end{array}$ & $\begin{array}{c}30,4 \pm 8,6 \\
(n=22)\end{array}$ & $\begin{array}{c}37,8 \pm 5,5 \\
(n=74)\end{array}$ \\
\hline \multirow[t]{2}{*}{$\begin{array}{l}\text { OLRI G501C } \\
(G C)\end{array}$} & $\begin{array}{c}\text { СД2 + ОП } \\
(n=64)\end{array}$ & $\begin{array}{l}19,3 \pm 5,2 \\
(n=56)^{1}\end{array}$ & $0(n=8)$ & $\begin{array}{c}17,9 \pm 5,1 \\
(n=55)\end{array}$ & $\begin{array}{c}11,1 \pm 10,2 \\
(n=9)\end{array}$ & $\begin{array}{c}23,1 \pm 11,5 \\
(n=12)\end{array}$ & $\begin{array}{c}15,4 \pm 4,8 \\
(n=52)\end{array}$ \\
\hline & $\begin{array}{l}\text { СД2, все } \\
(n=96)\end{array}$ & $\begin{array}{l}20,9 \pm 4,3 \\
(n=85)^{1}\end{array}$ & $0(n=11)$ & $\begin{array}{l}20,5 \pm 4,3 \\
(n=82)\end{array}$ & $\begin{array}{l}7,1 \pm 6,8 \\
(n=14)\end{array}$ & $\begin{array}{l}26,0 \pm 9,2 \\
(n=22)\end{array}$ & $\begin{array}{l}16,2 \pm 4,2 \\
(n=74)\end{array}$ \\
\hline \multirow[t]{2}{*}{$\begin{array}{l}L E P R G \ln 223 A r g \\
(A G)\end{array}$} & $\begin{array}{c}\text { СД2 + ОП } \\
(n=64)\end{array}$ & $\begin{array}{c}42,1 \pm 6,5 \\
(n=56)\end{array}$ & $\begin{array}{c}75,0 \pm 15,2 \\
(n=8)\end{array}$ & $\begin{array}{l}50,9 \pm 6,8 \\
(n=55)\end{array}$ & $\begin{array}{c}22,2 \pm 13,8 \\
(n=9)\end{array}$ & $\begin{array}{c}33,3 \pm 13,6 \\
(n=12)\end{array}$ & $\begin{array}{c}50,0 \pm 6,9 \\
(n=52)\end{array}$ \\
\hline & $\begin{array}{c}\text { СД2, все } \\
(n=96)\end{array}$ & $\begin{array}{l}46,5 \pm 5,4 \\
(n=85)^{1}\end{array}$ & $\begin{array}{c}81,8 \pm 11,5 \\
(n=11)\end{array}$ & $\begin{array}{c}51,8 \pm 5,4 \\
(n=82)\end{array}$ & $\begin{array}{c}42,8 \pm 13,2 \\
(n=14)\end{array}$ & $\begin{array}{c}39,1 \pm 10,2 \\
(n=22)\end{array}$ & $\begin{array}{c}54,1 \pm 5,9 \\
(n=74)\end{array}$ \\
\hline \multirow[t]{2}{*}{$\begin{array}{l}S H B G, r s 6257 \\
(T C)\end{array}$} & $\begin{array}{c}\text { СД2 + ОП } \\
(n=64)\end{array}$ & $\begin{array}{c}16,0 \pm 4,8 \\
(n=56)\end{array}$ & $\begin{array}{c}25,0 \pm 15,2 \\
(n=8)\end{array}$ & $\begin{array}{c}20,0 \pm 5,4 \\
(n=55)\end{array}$ & $0(n=9)$ & $\begin{array}{c}25,0 \pm 12,5 \\
(n=12)\end{array}$ & $\begin{array}{c}15,4 \pm 4,8 \\
(n=52)\end{array}$ \\
\hline & $\begin{array}{c}\text { СД2, все } \\
(n=96)\end{array}$ & $\begin{array}{c}15,3 \pm 3,9 \\
(n=85)\end{array}$ & $\begin{array}{c}18,2 \pm 11,5 \\
(n=11)\end{array}$ & $\begin{array}{l}18,3 \pm 4,1 \\
(n=82)^{2}\end{array}$ & $0(n=14)$ & $\begin{array}{c}18,2 \pm 8,1 \\
(n=22)\end{array}$ & $\begin{array}{c}14,9 \pm 4,1 \\
(n=74)\end{array}$ \\
\hline
\end{tabular}

Примечание. СД2 - сахарный диабет 2-го типа; ОП - онкопатология; СД2, все - объединенная группа больных сахарным диабетом 2 -го типа, имеющих или не имеющих онкологическое заболевание; $n-$ число наблюдений. ${ }^{1}-$ отличие от данных в группе ОСТ1 $\mathrm{R} 61 \mathrm{C}(-)$ достоверно $(p<0,03) ;^{2}$ - тенденция к отличию от данных в группе OCT1_rs622342 (-); ${ }^{3}-$ тенденция к отличию от данных в группе C11Orf65 (-). Дополнительная информация о степени выраженности различий представлена в разделе «Результаты».

больных диабетом генотип $A G$ рецепторов лептина $L E P R$ Gln 223Arg в метформинпозитивной подгруппе носителей полиморфизма OCT1_R61C встречался достоверно реже, чем в метформиннегативной $\left(\chi^{2}=4,71\right)$, что у больных с комбинацией «диабет + рак» было выражено в меньшей степени $\left(\chi^{2}=2,90\right.$; см. табл. 3$)$.

В случае носительства потенциально метформинпозитивных вариантов полиморфизма OCT1_R61C или OCT1_ rs622342 у больных с впервые диагностированным СД2 как с онкологическим заболеванием, так и без него отмечалось (на фоне отсутствия иных заметных различий изучавшихся параметров) достоверное увеличение индекса инсулинорезистентности по сравнению с носителями метформиннегативных генотипов $(5,18 \pm 0,74$ против $3,23 \pm 0,27$ усл. ед., $p=0,04$, при сочетании СД2 и онкопатологии и $5,29 \pm$ 0,77 против $3,31 \pm 0,66$ усл. ед, $p=0,05$, при заболевании только СД2, соответственно). Второй особенностью оказалась умеренная тенденция к снижению уровня эстрадиолемии, которая была присуща носителям метформинпозитивных вариантов, однако другой пары С-генов, а именно: STK11 $(63,7 \pm 14,2$ против $118,6 \pm 50,9$ пМ/л у метформиннегатив-

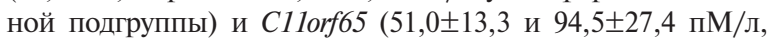
соответственно).

\section{Обсуждение}

Несмотря на многолетний интерес к метформину как к средству лечения СД и постепенное признание вариабельности и (не исключено) селективности его действия $[1,3,4]$, изучение фармакогенетики этого бигуанида еще не приобрело заметного размаха, а у онкологических больных, страдающих или не страдающих диабетом, ранее вовсе не проводилось. Между тем носительство однонуклеотидных полиморфизмов (SNPs), сопряженных с метаболизмом метформина или другими его свойствами, может рассматриваться как маркер и реального (что нуждается в дополнительном анализе [7]), и потенциального (как в настоящей работе) ответа на этот лекарственный препарат.

Полученные в исследовании результаты в своем большинстве отличаются новизной и заслуживают обсуждения в нескольких основных аспектах. В частности, выяснилось, что больные СД2, имеющие и не имеющие злокачественные новообразования, по генетическим критериям потенциальной чувствительности к метформину между собой отличаются в целом меньше, чем от онкологических больных без диабета, по пока 
не установленным причинам. Далее, оказалось, что отдельные полиморфизмы, характеризующие, по данным литературы [6-9], возможность ответа на метформин и отнесенные нами к числу «стандартных» (C), при сравнении их в одном исследовании могут выявляться в одних и тех же группах женщин с различной частотой (к примеру, представленность вариантов, казалось бы, родственных друг другу OCT1 R61C и OCT1 rs 622342; см. табл. 2). Соответственно, возможно, что для предсказания ответа на метформин в зависимости от обследуемой популяции окажется необходимым использовать не одинаковые, а различные фармакогенетические маркеры (в особенности при условии расширения их спектра). В этом отношении не стоит забывать о той пользе, которую в данном случае способно принести сочетанное использование «стандартных» и «ассоциированных» генетических маркеров, о чем свидетельствуют и результаты настоящей работы (см. табл. 3).

Обнаруженная более выраженная инсулинорезистентность у носителей метформинпозитивных полиморфных вариантов генов OCT1_R61C и OCT1_rs622342 представляется логичной и отчасти обсуждалась ранее [6]. В то же время отсутствие подобной находки у носителей аналогичных «по функции» вариантов генов STK11 и C11orf65, и напротив, обнаружение у них наклонности к гипоэстрогенемии, помимо иных возможных объяснений, напоминает о способности метформина не только к антидиабетическому, но и к другим эффектам, в частности, влиянию на эстрогенсинтетазную (ароматазную) активность [14]. Подобную точку зрения косвенно поддерживает и тот факт, что в объединенной группе больных СД2 и у страдающих диабетом онкологических больных с носительством некоторых потенциально метформинпозитивных стандартных генотипов, как оказалось, могут сочетаться определенные полиморфные варианты гена половые гормоны связывающего глобулина. По имеющимся данным, варианты этого гена вовлечены в модификацию как содержания свободного эстрадиола в крови, так и риска развития СД2 [10]. Такие наблюдения, как представляется, находятся в ряду факторов, «оправдывающих» сравнительный анализ фармакогенетических маркеров чувствительности к метформину одновременно с позиций его антиметаболического, эстрогенмодулирующего и антинеопластического действия, чему ранее внимания практически не уделялось.

\section{Заключение}

В итоге проведенного исследования установлено, что постменопаузальные больные сахарным диабетом 2-го типа, имеющие и не имеющие злокачественные новообразования, по генетическим критериям потенциальной чувствительности $\kappa$ метформину между собой отличаются в целом меньше, чем от онкологических больных без диабета, причины чего нуждаются в уточнении. Особенностью обследованных постменопаузальных женщин был тот факт, что диабет у многих из них был диагностирован впервые, что могло отразиться на нередко умеренном (по средним значениям) повышении содержания гликированного гемоглобина (данные не представлены). Другой особенностью стало относительно небольшое число пробандов в некоторых подгруппах, что заставляет рассматривать настоящее исследование как пилотное. Тем не менее результаты представляются важными, заслуживающими внимания и стимулируюшими дальнейшую работу в этом направлении, включая оценку не только потенциальных, но и реальных эффектов метформина в сопоставлении с результатами параллельно проводимого фармакогенетического или, по другой терминологии, фармакогеномного анализа.

Работа частично поддержана РФФИ (грант № 12-04-00084) и грантом Министерства образования и науки РФ (грант № 14.512.11.0041).

\section{REFERENCES}

1. Dedov I.I., Shestakova M.V. Sakharnyi diabet: diagnostika, lechenie, profilaktika [Diabetes: Diagnostics, Treatment, Prevention]. Moscow, MIA, 2011. $803 \mathrm{p}$.

2. Giovannucci E., Harlan D.M., Archer M.C., Bergenstal R.M., Gapstur S.M., Habel L.A., Pollak M., Regensteiner J.G., Yee D. Diabetes and cancer: a consensus report. Diabetes Care. 2010; 33 (7): 1674-1685.

3. Bershtein L.M. Diabetes mellitus - Diabetes mellitus. 2012; 4: 81-88.

4. Pollak M.N. Investigating metformin for cancer prevention and treatment: the end of the beginning. Cancer Discov. 2012; 2 (9): 778-790.

5. Ong C.R., Molyneaux L.M., Constantino M.I., Twigg S.M., Yue D.K. Long-term efficacy of metformin therapy in nonobese individuals with type 2 diabetes. Diabetes Care. 2006; 29 (10): 2361-2364.

6. Becker M.L., Visser L.E., van Schaik R.H., Hofman A., Uitterlinden A.G., Stricker B.H. Genetic variation in the organic cation transporter 1 is associated with metformin response in patients with diabetes mellitus. Pharmacogenomics J. 2009; 9 (4): 242-247.

7. Zolk O. Disposition of metformin: variability due to polymorphisms of organic cation transporters. Ann. Med. 2012; 44 (2): 119-129.

8. GoDARTS and UKPDS Diabetes Pharmacogenetics Study Group; Wellcome Trust Case Control Consortium 2, Zhou K. Bellenguez C., Spencer C.C. et al. Common variants near ATM are associated with glycemic response to metformin in type 2 diabetes. Nat. Genet. 2011; 43 (2): 117-120.

9. Legro R.S., Barnhart H.X., Schlaff W.D., Carr B.R., Diamond M.P., Carson S.A., Steinkampf M.P. et al. Ovulatory response to treatment of polycystic ovary syndrome is associated with a polymorphism in the STK11 gene. J. Clin. Endocrinol. Metab. 2008; 93 (3):792800 .

10. Le T.N., Nestler J.E., Strauss J.F. 3rd, Wickham E.P. Sex hormonebinding globulin and type 2 diabetes mellitus. Trends Endocrinol. Metab. 2012; 23 (1): 32-40.

11. Thameem F., Puppala S., Lehman D.M., Stern M.P., Blangero J., Abboud H.E., Duggirala R., Habib S.L. The $\operatorname{Ser}(326)$ Cys polymorphism of 8-oxoguanine glycosylase 1 (OGG1) is associated with type 2 diabetes in Mexican Americans. Hum. Hered. 2010; 70 (2): $97-101$.

12. Ulybina Yu.M., Imyanitov E.N., Vasil'ev D.A., Bershtein L.M. Mol. boil - Molecular biology. 2008; 42(6): 947-956.

13. Matthews D.R., Hosker J.P., Rudenski A.S., Naylor B.A., Treacher D.F., Turner R.C. Homeostasis model assessment: insulin resistance and beta-cell function from fasting plasma glucose and insulin concentrations in man. Diabetologia. 1985; 28 (7): $412-419$.

14. Samarajeewa N.U., Ham S., Yang F., Simpson E.R., Brown K.A. Promoter-specific effects of metformin on aromatase transcript expression. Steroids. 2011; 76 (8): 768-771. 


\section{FOR CORRESPONDENCE}

Bershtein Lev Mikhailovich, PhD, professor, Head of the Laboratory of Oncoendocrinology of FSBI "N.N. Petrov Research Institute of Oncology".

MD, professor, head of Laboratory of Oncoendocrinology, Federal State Budget Institution "N.N. Petrov Scientific Research Institute of Oncology"

Address: 68, Leningradskaya Street, Pesochnii, St. Petersburg, RF, 197758; tel.: +7 (812) 439-9536,

e-mail: levmb@endocrin.spb.ru

Ievleva Aglaya Gennadievna, MD, research scientist of Molecular Oncology Laboratory of FSBI "N.N. Petrov Research Institute of Oncology".

Address: 68, Leningradskaya Street, Pesochnii, St. Petersburg, RF, 197758; tel.: +7 (812) 439-9528; e-mail: aglayai@inbox.ru Vasil'ev Dmitrii Alekseevich, MD, senior research scientist of Oncoendocrinology Laboratory of FSBI "N.N. Petrov Research Institute of Oncology".

Address: 68, Leningradskaya Street, Pesochnii, St. Petersburg, RF, 197758; tel.: +7 (812) 439-9536;

e-mail: dvasilyev@hotmail.com

Kovalenko Irina Mikhailovna, research scientist of Oncoendocrinology Laboratory of FSBI "N.N. Petrov Research Institute of Oncology".

Address: 68, Leningradskaya Street, Pesochnii, St. Petersburg, RF, 197758; tel.: +7 (812) 439-9536; e-mail: ira_dv@mail.ru Imyanitov Evgenii Naumovich, PhD, professor, Head of the Department of Tumor Growth Biology of FSBI "N.N. Petrov Research Institute of Oncology".

Address: 68, Leningradskaya Street, Pesochnii, St. Petersburg, RF, 197758; tel.: +7 (812) 439-9528;

e-mail: evgeny@imyanitov.spb.ru 